\title{
Combined method of calculation of the residual life of building structures
}

\author{
Marina Gravit ${ }^{1}$, Dmitry Korolkov ${ }^{1 *}$ and Irina Reshetnikova ${ }^{2}$ \\ ${ }^{1}$ Peter the Great St. Petersburg Polytechnic University, 29 Politechnicheskaya St., St. Petersburg, \\ 195251, Russia \\ ${ }^{2}$ Tyumen Industrial University, Volodarskogo str., 38, Tyumen, 625000, Russia
}

\begin{abstract}
This article presents some methods for calculating the residual life of building structures. Areas of application for each of them are highlighted. A new method for calculating the residual life of building structures is proposed, which is proposed to be called a combined procedure for calculating the residual life of building structures. This method outlines a methodical approach that involves the joint use of all the techniques listed in this article. This will remove the limitations that each of these techniques had individually, which makes the combined methodology a universal technique for calculating the residual life of building structures. Combined methodology with its further improvement allows the use in some cases of special methods for calculating the remaining resource.
\end{abstract}

\section{Introduction}

Due to the fact that there are ways to calculate the remaining resource, you can get a reasonable idea of how much the building or structure can still be operated. In some cases, this is of great importance in terms of economics.

The purpose of this article will be to consider some of the techniques for calculating the residual resource of both Russian [1,2] and foreign researchers [11-21]. The limitations of each of these techniques will be shown and a combined methodology for calculating the remaining resource is presented, combining all of the listed techniques. This will eliminate the shortcomings of each of these techniques separately.

\section{Methods}

\section{Method for calculating the residual resource by the Gaussian distribution}

Determination of the residual life is made using curves of physical wear of building structures, depending on the terms of their operation, given in $[3,4]$ for building structures. Based on the data of the technical survey on the graphs given in fig. 1, the "actual age" of the elements of the supporting framework of an isotope laboratory is determined, after which a

\footnotetext{
*Corresponding author: korol9520@yandex.ru
} 
probable (final) calculation of the reliability of building structures is made with a corresponding adjustment and an additional life of the building structures is determined.

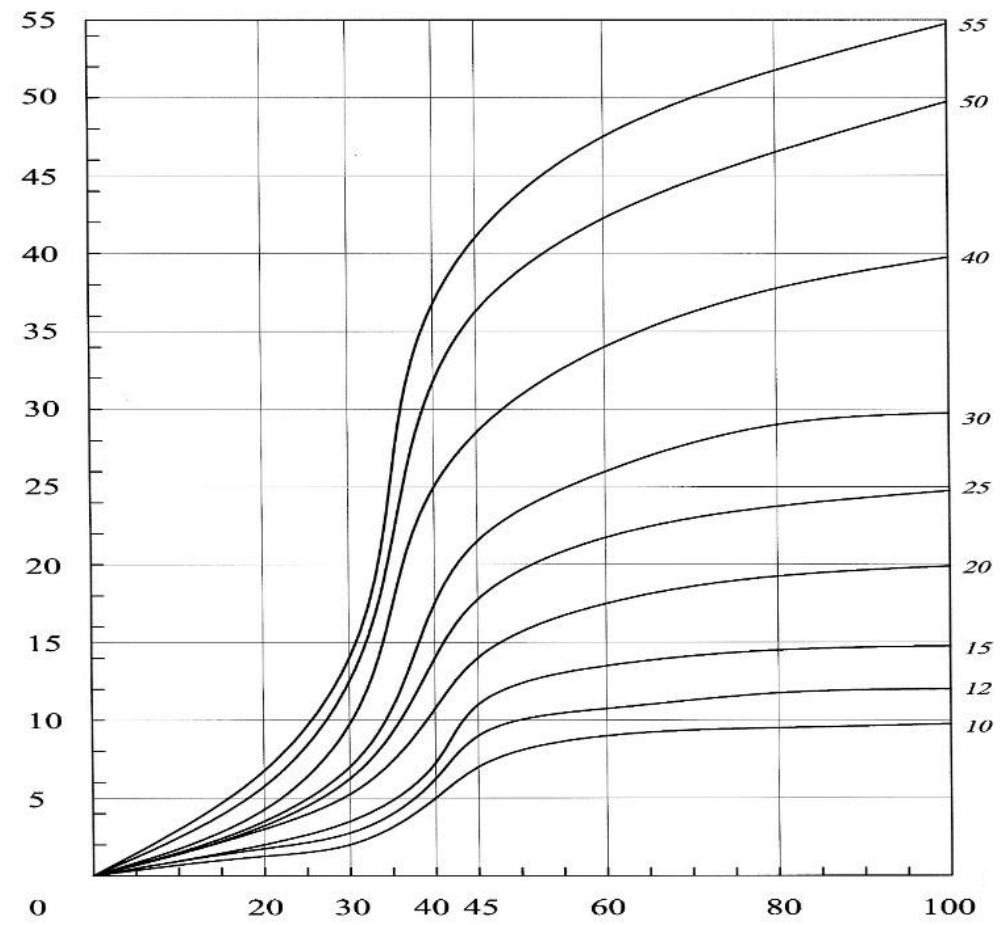

Fig. 1. Dependence of physical wear of elements of building structures on the life of the facility (with the service life of objects equal to 10-55 years).

After all the initial data are determined: the average lifetime of the element, the standard deviation of the lifetime of the element from its mean value, the significance coefficients, the category of TC (wear), the actual age of the elements along the curves of physical wear, the probability of failure-free operation is calculated.

The probability of failure-free operation is given by the formula:

$$
\begin{gathered}
P(x)=\frac{1}{\sqrt{2 \pi}} \int_{\infty}^{x} e^{-\frac{z^{2}}{2}} d z \\
x=\frac{T_{x}-t}{G x}
\end{gathered}
$$

$$
\mathrm{G}_{\mathrm{x}} \approx 0.18 \times \mathrm{T}_{\mathrm{x}} .
$$

Such a calculation is greatly simplified by the fact that there are tables of values of this function as a function of the variable $\mathrm{x}$. The total probability of failure-free operation of all building structures can be considered in many ways. The first is finding the arithmetic mean by summing up the probabilities of failure-free operation of all building structures (elements). Using this approach, an overestimated probability of failure-free operation is obtained due to the fact that all elements are considered to be "equal in rights" and low-value elements 
(having low weight (significance factor)) contribute increasing their high probability. The second, as the total probability, is the smallest of the probabilities of the element. The advantage of this approach is that we are guaranteed to get an estimate below the true one, which means that the assessment will always be reliable. The disadvantage is that we seem to underestimate the residual resource. Third, the approach is that we summarize the probability of failure-free operation of the elements, multiplying by their significance factor. With this approach, we get the value closest to the true probability of failure-free operation.

This technique has one significant drawback. It can only be applied to some metal building structures. This is due to the fact that at that time, metal structures were not as widespread in construction as now. After the release of [4], in fact, no studies were conducted to determine the dependence of physical wear on the age of the structures. Therefore, due to the lack of such data, this technique has limitations regarding metal structures.

It is also worth noting that this technique has limitations in the field of application. This restriction is imposed by virtue of the fact that the assumption of the normal distribution law (Gaussian distribution law) of the probability of failure-free operation of building structures is introduced.

Restrictions can be formulated as follows:

1. The technique is applied only to structures having a long service life and / or not operating in an aggressive environment.

For constructions that have a short lifespan, this technique is undesirable, because it gives an overestimate of the residual resource. This is due to the fact that such structures wear out quickly enough and the Poisson distribution is most suitable for describing the process of physical wear. Analogous arguments for the structures working in an aggressive environment.

2. The methodology does not apply to buildings and structures that have a short lifespan.

This mainly applies to temporary structures. This is due to their functional purpose and, consequently, this causes the use of cheap materials with a short service life when they are built.

\section{Method for determination of residual life of metal structures}

One of the drawbacks of the methodology presented above was that it did not allow calculating the residual life of metal structures. To solve this problem, one can use the technique used to determine the residual life of metal pipelines that are used in the nuclear field [7], [8] and the oil and gas sector.

This definition of the remaining resource by "thinning" the wall. It is assumed that during the operation of the pipelines, they corrode for some thickness of the pipe wall. If you clean the metal surface of the pipe from corrosion, then you can measure the actual wall thickness. If it turns out to be more than the minimum allowable (established by the requirements of regulatory documentation or under the project), we get a "stock". Knowing the rate of corrosion, we can calculate the remaining life of this pipeline.

The essence of the methodology is as follows.

Determination of the remaining life of pipelines is based on the analysis and calculation of the strength of the most stressed sections of pipelines.

During the operation period, the thinning of the wall of the most loaded section of the pipeline was

$$
s_{f}=S-S_{f}
$$

where S - the effective thickness of the wall, mm (selected from the design (design) documentation);

$S_{f}$ - is the actual wall thickness, mm (obtained from the pipeline survey);

$\mathrm{sf}$ - actual wall thinning during the operation period, $\mathrm{mm}$. 
Accepting (conservatively) the linear law of the time variation of the wall thickness of the most loaded pipeline section caused by the medium, we obtain the value of thinning for a general period (including the prolonged period).

It should be noted that one can also use the nonlinear law of time variation of the wall thickness.

But to apply it you need to have a good base of observations of the corrosion process, which is quite rare. Therefore, it is better to use a linear law.

The average wall thinning speed is:

$$
v=\frac{S+C_{o}-S_{f}}{t}
$$

$\mathrm{C}_{\mathrm{o}}$ - plus tolerance for wall thickness, $\mathrm{mm}$;

$\mathrm{t}$ - is the time from the start of operation to the time of the examination, year.

Then the permissible wall thickness Sdop will be reached through:

$$
T=\frac{S_{f}-S_{O}}{v}
$$

where $\mathrm{T}$ - is the period during which the pipeline will remain operational, years;

This principle of calculating the residual life of metal structures is used in construction in a somewhat truncated form [9], [10].

There are a number of problems in this approach.

The first problem, the accuracy of determining the actual thickness of the metal structure, not damaged by corrosion. Depending on the method of measurement and the availability of the design, the error can reach several centimeters. In general, accessibility is the determining factor in the accuracy of the measurement, because to determine the thickness of the structure, not damaged by corrosion, it must first be cleaned, and this is done or can be done not always.

The second problem is determining the minimum thickness of the structure. Here, the minimal thickness of the construction is understood as the minimum geometric dimension, which is ensured by the perception of a given force. You can define it in different ways.

First, you can take this size from the project documentation. When calculating the building structure, the dimensions are chosen there, based on the condition of perception of the given forces. This is the most simple and inexpensive way, because it does not require additional work to determine the minimum thickness of the structure. But in this case there is no guarantee that the presented minimum sizes will correspond to reality. There may be several reasons for this:

- the construction takes on a different load;

- the scheme of design work has changed;

- in the production of construction works, changes were made that were not reflected in the project, etc.

Therefore, project documentation is not entirely reliable source of information. In addition, in practice, project documentation is often lost.

Secondly, it is possible to determine the minimum size by conducting a detailed survey with the opening of building structures and performing a verification calculation. This is the most accurate way to determine the minimum thickness of a structure. True, this approach has certain drawbacks. It is time-consuming and expensive, so it is not always used.

In this technique, one limitation can be singled out, which appears when it is used to calculate "very thin" metal structures. These include, for example, sheets of corrugated board. Due to the fact that their thickness usually does not exceed $1 \mathrm{~mm}$ when the protective layers are broken, corrosion in fact in a short time affects the section of the element to the full depth. Therefore, there is no formally minimal wall thickness, and accordingly we can not calculate the residual resource.

Method for calculating the residual resource using the Poisson distribution. 
The overall damage assessment of the structure is made by the formula:

$$
\varepsilon=\frac{\alpha_{1} * \varepsilon_{1}+\alpha_{2} * \varepsilon_{2}+\cdots+\alpha_{i} * \varepsilon_{i}}{\alpha_{1}+\alpha_{1}+\cdots+\alpha_{i}}
$$

where $\varepsilon_{1}, \varepsilon_{2}, \ldots, \varepsilon_{\mathrm{i}}$ - the maximum damage to certain types of structures;

$\alpha_{1}, \alpha_{2}, \ldots, \alpha_{1}$. are the coefficients of significance of certain types of structures.

Relative evaluation of damages of the structure is made according to the formula [9], [10]:

$$
\gamma=1-\varepsilon
$$

The constant wear is determined from the survey data [9], [10]:

$$
\lambda=\frac{-\ln \gamma}{t_{\phi}}
$$

where $t_{\phi}$ is the service life in years at the time of the examination.

The service life of the building from the beginning of operation to the overhaul is determined by the formula in years [9], [10]:

$$
T=\frac{0,16}{\lambda}
$$

The coefficient of 0.16 corresponds to the probability of failure-free operation of 0.85 , which is referred to in [4] as a limited-operational state.

It is also worth noting that this technique has limitations in the field of application. This restriction is imposed by virtue of the fact that the assumption is made of the exponential distribution law (Poisson distribution law) of the probability of failure-free operation of building structures.

Restrictions can be formulated as follows:

1. The procedure is applied only to structures having a short service life and (or) operating under conditions of an aggressive environment.

This is due to the fact that this distribution has large quantiles, which lead to a rapid descending of the function. It is most suitable for materials and structures with a short service life, because they quickly wear out under the influence of external factors.

2. The methodology is applied to buildings and structures that have a short lifespan.

This mainly applies to temporary structures. This is due to their functional purpose and, consequently, this causes the use of cheap materials with a short service life when they are built.

Calculation of the remaining life for static strength

Residual resource by the criterion of the limiting state - the permissible voltage is [9], [10]:

$$
\mathrm{T}_{k}=\frac{\sigma_{b}(t)-[\sigma]}{\alpha_{\sigma}}
$$

where $\sigma_{b}(t)$ is the ultimate strength at the time of the survey;

$[\sigma]$ - ultimate strength by calculation;

$\alpha_{\sigma}$ - is the rate of decrease of mechanical properties.

The rate of decrease in mechanical properties [9], [10]:

where $\sigma_{b}$ - is the normative strength limit;

$$
\alpha_{\sigma}=\frac{\sigma_{b}-\sigma_{b}(t)}{t}
$$

$\mathrm{t}$ - is the time from the start of operation to the time of the survey.

This technique can only be used if a detailed (instrumental) survey of building structures is carried out. Moreover, the accuracy of this technique depends strongly on the method by which the strength of the material is determined. The greatest accuracy will be achieved when determining the strength by a press test.

Calculation of Residual Resource by Fatigue Structures

Resource cyclic performance is determined by the formula [9], [10]: 


$$
\mathrm{T}_{e}=\frac{\mathrm{T}_{e} *[N]}{N_{e}}
$$

where $\mathrm{T}_{\mathrm{e}}$ is the operating time from the start of operation, years;

$[\mathrm{N}]$ - allowable number of loading cycles;

$N_{e}$ - number of loading cycles for the period of operation.

Resource of residual capacity is determined by the formula [9], [10]:

$$
T_{\text {ost }(c)}=T_{c}-T_{e}
$$

This technique is suitable only for structures operating under dynamic loading conditions.

\section{Results and Discussion}

Since all the above methods for calculating the residual resource have limitations in the field of application, a combined methodology for calculating the residual life of building structures is proposed. Within the framework of this technique, the drawbacks that were inherent in the above methods are eliminated.

The procedure for calculating the remaining life of the proposed combined methodology for calculating the remaining life of building structures:

1. Determine the nomenclature of the elements for which the residual resource will be calculated.

2. Definition of operating conditions of a building or structure.

3. The choice of the method for calculating the residual life of building structures is made depending on the type of material, the type of construction, operating conditions, loading method, and so on.

The choice of the method for calculating the residual resource in the framework of the combined methodology for calculating the residual resource should be made, based on the following considerations.

If the building or structure is operated under normal conditions and (or) the service life is significant, that is, it is not a temporary structure, it is advisable to use the residual resource calculation method based on the law of normal distribution (Gaussian law). Exceptions also include metal structures and new modern building materials.

If the building or construction is operated under aggressive conditions and (or) the service life is insignificant, that is, it is a temporary structure, then it is advisable to use the residual resource calculation method based on the law of exponential distribution (Poisson distribution). Exceptions are also metal structures.

If the building or structure has metal structures, then the calculation of the remaining life should be made by "thinning" the wall.

If the design works under dynamic loads, then the calculation of the remaining life should be made by the method of calculating the remaining life of the fatigue of the structure. Moreover, this technique will have only an estimated (preliminary) nature of the residual resource. If necessary, the calculation of the residual life will be made by special methods that estimate the remaining life of the application only for these types of structures.

As a verification of the results of calculations using other methods, it is possible to calculate the residual life of building structures by the method of calculating the remaining life for static strength. Also, this technique is suitable for calculating the residual life of building structures made from new building materials.

Due to the use of the combined method of calculating the residual life of building structures, the limitations that the methods presented separately were eliminated, which makes the combined methodology for calculating the residual life of building structures universal. This technique can also be used to test or qualitatively evaluate the results obtained 
with the use of special techniques for calculating the residual life, designed for specific designs or conditions. The combined methodology can be used for various economic calculations. For example, by the amount of deductions to the capital repair fund or the feasibility of building a new facility instead of the one used.

\section{Conclusions}

A combined technique for calculating the residual life of building structures has been developed, which is universal and is suitable for calculating the residual life of building structures in all cases without exception.

The combined procedure for calculating the residual life of building structures can be improved by applying special techniques for calculating the residual life, designed for specific designs or conditions.

Also, this technique can be improved by taking into account the effect of failures for general reasons.

Also, this technique can be improved by taking into account failures that do not lead to failures of the entire system. This can be done by applying a common logical-probabilistic method and the corresponding software package.

\section{References}

1. M. Lazarevska, M., Cvetkovska, M., Knezevic, M., Trombeva Gavriloska, A., Milanovic, M., Murgul, V., Vatin, N. Applied Mechanics and Materials, 627, 276-282 (2014)

2. D. Korolkov, Proc.of the VIII International Scientific and Practical Conference. St. Petersburg. Publishing house Polytechnic, 98-108 (2017)

3. Russian State Standard MDS 13-14.2000

4. Russian State Standard VSN 53-86

5. Russian State Standard GOST 27751-2014

6. Russian State Standard GOST 31937-2011

7. Russian State Standard RD 03-421-01

8. СТ ЦКАБА 024-2006 Pipeline armature. Determining Residual Resource and Validity Reliability Valves

9. M. Permyakov, Calculation and evaluation of the residual resource of buildings (Magnitogorsk, Publishing house Magnitogorsk, 2011).

10. URL: http://www.paamag.ru/pressa/ostatokkresurs.

11. C.L. Su, L. Xin, et.al. Numerical Analysis of Ultimate Benging Capacity of Pipeline with Single Corrosion Defect. The 20th national structure engineering academic meeting. 360-367 (2011)

12. C.L. Wang, et.al. Journal of Sichuan University, 35(5), 50-54 (2003)

13. Unger D J. Path Independent Integral for an Elliptical Hole in a Plate under Tension for Plane Stress Deformation Theory. Journal of Elasticity. 2008, 92(3).217-226. 